\title{
Delivering better results for patients through core outcomes, measuring evidence and patient participation
}

Nasal obstruction is one of the most frequent and bothersome symptoms in rhinosinal disease ${ }^{(1-3)}$. Although we are able to measure nasal airflow, the correlation between the feeling of nasal obstruction and objective measurements has been shown to be average at most ${ }^{(4,5)}$. A significant proportion of the European population has a more or less deviated nasal septum and deviated septa have received a lot of attention from otorhinolaryngologists. In sharp contrast is the amount of hard evidence concerning septoplasty with or without turbinate surgery. In this issue of the Journal, van Egmond et al. ${ }^{(6)}$ systematically evaluate the effectiveness of septoplasty versus non-surgical management, and the addition of turbinate surgery versus septoplasty alone. They found no studies comparing septoplasty to non-surgical management, and for that reason a few years ago they started a large national trial with exactly that question in $\operatorname{mind}^{(7,8)}$. We eagerly await the results. The addition of turbinate surgery to septoplasty, evaluated in 11 papers, of which only 5 were RCTs showed no additional benefit. There was also no difference in objective and subjective benefits. The criteria for inclusion in this systematic review was nasal obstruction caused by septal deviation (in the eyes of the authors) without allergic mucosal disease unresponsive to treatment. Recently in this journal it was shown that both allergic and non-allergic patients gained benefit from surgery (turbinate surgery with or without septoplasty) to relieve nasal obstruction ${ }^{(1)}$. One of the papers included had 35/134 patients who also had nasal valve surgery ${ }^{(7)}$. Two studies indicated that patients with nasal valve problems were excluded ${ }^{(7)}$. Recently, a very interesting paper evaluating the cause of nasal obstruction in a large cohort of 1906 patients showed $2 / 3$ of the patients to have nasal valve collapse, and $82 \%$ percent of the 236 patients with severe/extreme NOSE scores who reported prior septoplasty and/or inferior turbinate reduction had nasal valve collapse ${ }^{(9)}$. In this group surgery is not always necessary; the Leuven group reported very positive results and acceptance of nasal valve dilatators ${ }^{(10)}$. The Rhinologic Society has spent a lot of time and effort on evaluating the evidence for our daily practice ${ }^{(11-17)}$. An important outcome of these analyses is the lack of strong evidence for many aspects of our daily clinical care. This is especially true in evaluation of surgery, and septal surgery does not seem to be an exception. It has proven difficult to carry out randomized trials ${ }^{(18)}$ and we are still struggling as to how to combine other forms of evidence, such as large cohort studies ${ }^{(1,9,19,20)}$, real-life studies $^{(13,21,22)}$ and e-studies ${ }^{(23-26)}$ to deliver the best possible care for our patients. Moreover, we have not always been able to decide among us what the most relevant outcome measures are for different diseases ${ }^{(27)}$. Quite often we still encounter unvalidated outcome measures, such as SNOT scores used in rhinitis, or validated outcome measures still have to be developed ${ }^{(28,29)}$. For rhinosinusitis, an outcome measure set was recently developed using an e-Delphi process allowing 110 patients and healthcare practitioners to individually rate outcomes in terms of importance and core outcomes for trials of interventions: CHronic Rhinosinusitis Outcome MEasures (CHROME) ${ }^{(12,30)}$. The set can be freely downloaded from the rhinology or ERS website (www. rhinology.com). It would be good if these outcome sets were also developed for other diseases in rhinology such as AR and NAR, and maybe also related to interventions like septoplasty. When we have gathered all the available evidence we need to incorporate it in our daily patient care. Guidelines and position papers are easy and renowned ways to update our knowledge in daily practice ${ }^{(31-33)}$. We now want to include the available knowledge in our consultations with patients, and using shared decision making ${ }^{(34)}$ find the best management for the disease of individual patients. However, we are more and more aware that evidence collected on groups of patients with more or less equal phenotype, e.g. patients with CRSwNP, might not be applicable to all patients in that group ${ }^{(35)}$. We do realize that we need precision medicine to optimize patient care ${ }^{(36,37)}$. The combination of personalized care by implementing endotyping ${ }^{(38-42)}$. Prediction of treatment success ${ }^{(43,44)}$ and deciding about the best moment to start a certain treatment ${ }^{(43,44)}$, prevention of disease $^{(45-47)}$ and patient participation in the elaboration of the treatment plan $^{(48)}$ is expected to substantially improve the therapeutic approach for individuals suffering from chronic disabling conditions. In this issue of the journal, we report on the use of mySinusitisCoach, an excellent way to involve patients with chronic rhinosinusitis in the management of their disease using mobile technology ${ }^{(48)}$. The use of mySinusitisCoach facilitates evaluation of patient reported outcome measures and allows not only tailoring of treatment to the level of control of the disease but will also allow us in future to collect longitudinal data about our patient care. This issue of the journal is full of very interesting papers about different subjects; from cystic fibrosis $^{(49,50)}$ to autonomic disfunction ${ }^{(51)}$, and from calvarian bone for the reconstruction of frontal defects ${ }^{(52)}$ to upregulation of TRPV1 expression in chronic rhinosinusitis with nasal polyps ${ }^{(53)}$. I wish you all a wonderful summer, with lots of sunshine. 
1. Parthasarathi K, et al. Airflow and symptom outcomes between allergic and non-allergic rhinitis patients from turbinoplasty. Rhinology. 2017:55(4):332-8.

2. Dietz de Loos DA, et al. Symptoms in chronic rhinosinusitis with and without nasal polyps. Laryngoscope. 2013;123(1):57-63.

3. Hirsch $A G$, et al. Nasal and sinus symptoms and chronic rhinosinusitis in a population-based sample. Allergy. 2017;72(2):274-81.

4. Verhoeven S, Schmelzer B. Type and severity of septal deviation are not related with the degree of subjective nasal obstruction. Rhinology. 2016;54(4):355-60.

5. Pendolino $\mathrm{AL}$, et al. Comparison between unilat eral PNIF and rhinomanometry in the evaluation of nasal cycle. Rhinology. 2018;56(2):122-6.

6. van Egmond $M$, et al. Septoplasty for nasal obstruction due to a deviated nasal septum in adults: a systematic review. Rhinology. 2018;56(3): 195-208.

7. van Egmond MM, et al. Effectiveness of septoplasty versus non-surgical management for nasal obstruction due to a deviated nasal septum in adults: study protocol for a randomized controlled trial. Trials. 2015;16:500.

8. van Egmond $\mathrm{M}$, et al. Clinimetric properties of the Glasgow Health Status Inventory, Glasgow Benefit Inventory, Peak Nasal Inspiratory Flow, and 4-Phase Rhinomanometry in adults with nasal obstruction. Rhinology. 2017;55(2):126-34.

9. Clark DW, et al. Nasal airway obstruction: Prevalence and anatomic contributors. Ear, nose, \& throat journal. 2018;97(6):173-6

10. Lekakis $\mathrm{G}$, et al. Managing nasal valve compromise patients with nasal dilators: objective vs. subjec tive parameters. Rhinology. 2016;54(4):348-54

11. Dhami $\mathrm{S}$, et al. Allergen immunotherapy for allergic rhinoconjunctivitis: A systematic review and meta-analysis. Allergy. 2017;72(11):1597-631.

12. Hopkins $C$, et al. Identifying the most important outcomes for systematic reviews of interventions for rhinosinusitis in adults: working with Patients, Public and Practitioners. Rhinology. 2016;54:20-6.

13. Lal $D$, et al. Management of rhinosinusitis during pregnancy: systematic review and expert pane recommendations. Rhinology. 2016;54(2):99-104.

14. Leason SR, et al. Association of gastro-oesophageal reflux and chronic rhinosinusitis: systematic review and meta-analysis. Rhinology. 2017:55:3-16.

15. Musgrave KM, Powell J. A systematic review of anti-thrombotic therapy in epistaxis. Rhinology. 2016;54(4):292-391.

16. Winblad $L$, et al. The risk of osteoporosis in ora steroid treatment for nasal polyposis: a systematic review. Rhinology. 2017:55(3):195-201.

17. Pundir $\mathrm{V}$, et al. Role of corticosteroids in Functional Endoscopic Sinus Surgery--a systematic review and meta-analysis. Rhinology. 2016;54(1):3-19.

18. Blencowe NS, et al. Interventions in randomised controlled trials in surgery: issues to consider during trial design. Trials. 2015;16:392

19. Erskine $\mathrm{S}$, et al. A cross sectional analysis of a casecontrol study about quality of life in CRS in the UK a comparison between CRS subtypes. Rhinology. 2016:54(4):311-5

20. Godoy SJ, Godoy JM, Godoy A, Revelo C. A straight choice: avoiding septal re-deviation using titanium plates. A 16 year retrospective patien follow-up evaluation. Rhinology. 2017;55(4):369-

21. Droessaert $V$, et al. Real-life study showing better control of allergic rhinitis by immunotherapy than regular pharmacotherapy. Rhinology. 2016;54(3):214-20.

22. van der Veen J, et al. Real-life study showing uncontrolled rhinosinusitis after sinus surgery in a tertiary referral centre. Allergy. 2017;72(2):282-90

23. Bousquet J, et al. Work productivity in rhiniti using cell phones: The MASK pilot study. Allergy. 2017:72(10):1475-84

24. Bousquet J, et al. Pilot study of mobile phone technology in allergic rhinitis in European countries: the MASK-rhinitis study. Allergy. 2017:72(6):857-65.

25. Bousquet J, et al. Daily allergic multimorbidity in rhinitis using mobile technology: A novel concept of the MASK study. Allergy. 2018.

26. Bousquet J, et al. Treatment of allergic rhinitis using mobile technology with real-world data: The MASK observational pilot study. Allergy. 2018.

27. Ottaviano G, Fokkens WJ. Measurements of nasa airflow and patency: a critical review with emphasis on the use of peak nasal inspiratory flow in daily practice. Allergy. 2016;71(2):162-74

28. Surda $\mathrm{P}$, et al. Rhinitis and its impact on quality of life in swimmers. Allergy. 2018;73(5):1022-31.

29. Segboer $C L$, et al. Quality of life is significantly impaired in nonallergic rhinitis patients. Allergy. 2018:73(5):1094-100.

30. Hopkins C, et al. CHronic Rhinosinusitis Outcome MEasures (CHROME), developing a core outcome set for trials of interventions in chronic rhinosinusitis. Rhinology. 2018;56(1):22-32.

31. Hummel T, et al. Position paper on olfactory dys function. Rhinol Suppl. 2017 Mar;54(26):1-30.

32. Hellings PW, et al. Non-allergic rhinitis: Position paper of the European Academy of Allergy and Clinical Immunology. Allergy. 2017;72(11):1657-65.

33. Sorokowska $A$, et al. Effects of olfactory training: a meta-analysis. Rhinology. 2017;55(1):17-26

34. Ikeda AK, et al. Evidence-Based Medicine in Otolaryngology Part 7: Introduction to Shared Decision Making. Otolaryngol Head Neck Surg. 2018;158(4):586-93.

35. Fokkens WJ. Evidence-based and precision medicine two of a kind. Rhinology. 2017;55(1):1-2.

36. Hellings PW, et al. Positioning the principles of precision medicine in care pathways for allergic rhinitis and chronic rhinosinusitis - A EUFOREAARIA-EPOS-AIRWAYS ICP statement. Allergy. 2017;72(9):1297-305

37. Fokkens WJ, et al. Rhinology Future Debates, an EUFOREA Report. Rhinology. 2017;55(4):298-304

38. Liao B, et al. Multidimensional endotypes of chronic rhinosinusitis and their association with treatment outcomes. Allergy. 2018;73(7):1459-69.

39. Zissler UM, et al. Current and future biomarkers in allergic asthma. Allergy. 2016;71(4):475-94.

40. Papadopoulos NG, et al. Phenotypes and endotypes of rhinitis and their impact on management: a PRACTALL report. Allergy. 2015;70:474-94.

41. Wei $B$, et al. Multivariate analysis of inflammatory endotypes in recurrent nasal polyposis in a Chinese population. Rhinology. 2018;56(3):

42. De Greve G, et al. Endotype-driven treatment in chronic upper airway diseases. Clin Translat Allergy. 2017;7:22
43. Rudmik $L$, et al. Defining appropriateness criteria for endoscopic sinus surgery during management of uncomplicated adult chronic rhinosinusitis: a RAND/UCLA appropriateness study. Rhinology. 2016;54(2):117-28

44. Rudmik L, et al. Using postoperative SNOT-22 to help predict the probability of revision sinus surgery. Rhinology. 2016:54(2):111-6.

45. Hopkins $C$, et al. Does time to endoscopic sinus surgery impact outcomes in Chronic Rhinosinusitis? Prospective findings from the National Comparative Audit of Surgery for Nasal Polyposis and Chronic Rhinosinusitis. Rhinology. 2015:53(1):10-7.

46. Hopkins $C$, et al. Does time to endoscopic sinus surgery impact outcomes in chronic rhinosinusitis? Retrospective analysis using the UK clinical practice research data. Rhinology. 2015;53(1):1824.

47. Schlosser RJ, et al. Asthma quality of life and control after sinus surgery in patients with chronic rhinosinusitis. Allergy. 2017;72(3):483-91.

48. Seys SF, et al. mySinusitisCoach: patient empowerment in chronic rhinosinusitis using mobile technology. Rhinology. 2018:56(3): 209-215.

49. Manji J, et al. The presence of Interleukin-13 in nasal lavage may be a predictor of nasal polyposis in pediatric patients with cystic fibrosis. Rhinology 2018:56(3): 261-267.

50. Aanaes $\mathrm{K}$, et al. The accessibility of topical treat ment in the paranasal sinuses on operated cystic fibrosis patients assessed by scintigraphy. Rhinology 2018:56(3): 268-273.

51. Chen WC, et al. The symptom burden of autonomic dysfunction is positively associated with chronic rhinosinusitis status. Rhinology 2018;56(3):227-233.

52. Dova S, et al. Reconstruction of frontal defects with calvarial grafts. Rhinology 2018;56(3):297-302

53. Toth $E$, et al. Upregulation of extraneuronal TRPV expression in chronic rhinosinusitis with nasal polyps. Rhinology. 2018;56(3):245-254

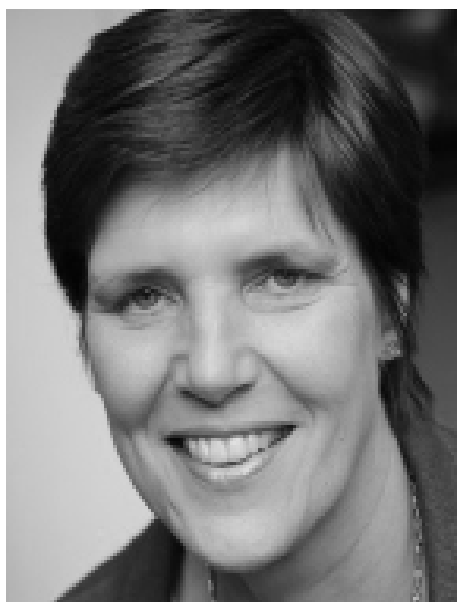

Wytske J. Fokkens, Editor-in Chief Amsterdam, the Netherlands 